UCRL-JC-126828

PREPRINT

\title{
Concept for High-Charge-State Ion Induction Accelerators
}

\author{
B. G. Logan, M. D. Perry, \\ and G. J. Caporaso
}

This paper was prepared for submittal to International Atomic Energy Agency Technical Committee Meeting on Drivers and Ignition Facilities for Inertial Fusion

Osaka, Japan

March 10-14, 1997

November 15, 1996

This is a preprint of a paper intended for publication in a journal or proceedings. Since changes may be made before publication, this preprint is made available with the understanding that it will not be cited or reproduced without the permission of the author. 


\section{DISCLAIMER}

This document was prepared as an account of work sponsored by an agency of the United States Government. Neither the United States Government nor the University of California nor any of their employees, makes any warranty, express or implied, or assumes any legal liability or responsibility for the accuracy, completeness, or usefulness of any information, apparatus, product, or process disclosed, or represents that its use would not infringe privately owned rights. Reference herein to any specific commercial product, process, or service by trade name, trademark, manufacturer, or otherwise, does not necessarily constitute or imply its endorsement, recommendation, or favoring by the United States Government or the University of California. The views and opinions of authors expressed herein do not necessarily state or reflect those of the United States Government or the University of California, and shall not be used for advertising or product endorsement purposes. 


\title{
Concept for High-Charge-State Ion Induction Accelerators
}

\author{
B. Grant Logan, Michael D. Perry, and George J. Caporaso \\ Lawrence Livermore National Laboratory * \\ L-481 Livermore, California, USA, 94550 \\ IAEA TCM on Drivers and Ignition Facilities for Inertial Fusion \\ Osaka University, Osaka, Japan, March 10-14 1997
}

\begin{abstract}
This work describes a particular concept for ion induction linac accelerators using highcharge-state ions produced by an intense, short pulse laser, and compares the costs of a modular driver system producing $6.5 \mathrm{MJ}$ for a variety of ion masses and charge states using a simple but consistent cost model.
\end{abstract}

\section{Introduction and concept}

The HIFSA study [1] found significant cost savings using heavy-ions of charge state $q=3$ relative to $\mathrm{q}=1$, but further pursuit of high charge state ion designs was discouraged primarily because (a) beam transport costs in the low energy "front end" of the accelerator, which the HIFSA study had neglected, was expected to be expensive, (b) existing high-q ion sources, including laser-plasma sources [2], lacked sufficient charge-state purity, and (c), higher space charge with high-q ions would increase the minimum focal spot size or number of beams required in high-vacuum target chambers. Since the HIFSA study, PIC calculations [3] have shown that a few percent ionization of the low pressure background vapor $\left(\sim 10^{-3}\right.$ torr $)$ present in an fusion chamber is sufficient to virtually eliminate the effects of beam space-charge on focal spot size.

The advent of intense, ultra-short-pulse lasers now suggests that specific high-ion charge states can be produced by multi-photon absorption at a controlled laser intensity, and with minimal collateral collisional ionization [4]. The laser-ion source concept schematically shown in Fig. 1 is proposed to address the issues (a) and (b) above. A frozen or liquid pellet of noble gas such as Argon or Xenon with the desired number of ions $\left(\mathrm{N}_{\mathrm{io}} \sim 10^{14}\right.$ to $10^{15}$ for a full-scale driver pulse) is first injected into a diode gap near the small anode end, when the injector voltage is initially off. A low energy laser pre-pulse shocks the 5 to 10 micron-radius pellet into a gas which is allowed to expand until a desired density $\left(10^{-5}\right.$ to $10^{-6} \mathrm{x}$ critical density) is reached within the main laser focal spot. The initial density must be sufficiently low that recombination does not occur subsequent to the main laser pulse before the ions are extracted. An intense, ultra-short main laser pulse $(\sim 100 \mathrm{fs})$ then strips the ions down to a common ionization level by multiphoton absorption that is controlled by the laser intensity $\left(10^{15}\right.$ to $10^{17} \mathrm{~W} / \mathrm{cm}^{2}$ for ionization levels near 0.2 to $1 \mathrm{keV}$, depending on the laser wavelength and charge state). The diode voltage is then erected within $20 \mathrm{~ns}$ after the laser plasma begins to expand after the main laser pulse. Ions are extracted from the plasma sheath during expansion until the ion density is depleted.

The pulse length is proportional to the initial number of ions in the pellet, and inversely proportional to the space-charge-limited extraction current that is constrained by the diode gap size and voltage. The diode gap size is chosen so that the laser-plasma sheath expansion during the extraction pulse does not exceed $10 \%$ of the initial diode gap. A double solenoid is used to provide control over the shape and extent of the solenoid fringe field in the gap, so as to provide a matched Brillouin flow beam during the flat-top part of the diode voltage pulse. 
Ions extracted

from spherically

expanding

plasma sheath

$a_{\mathrm{s}} \sim 10 \mathrm{~cm}, 1 \mu \mathrm{s}$

Injected pellets

initially shocked

to $\sim 0.01$ critical

$\mathrm{n}_{\mathrm{e}} @ \mathrm{r}_{\mathrm{o}} \sim 100 \mu \mathrm{m}$

$w /$ laser prepulse

Anode @ $\phi=+V_{s}$ electrons (a) Laser-ion injector

$0.4 \mu \mathrm{m}$,

$\sim 0.1 \mathrm{ps}$

laser

$\downarrow \downarrow$

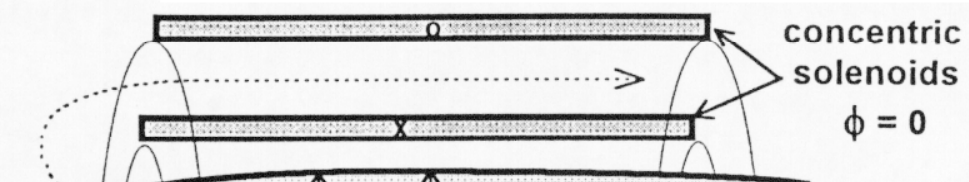

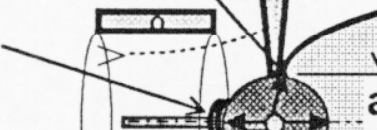
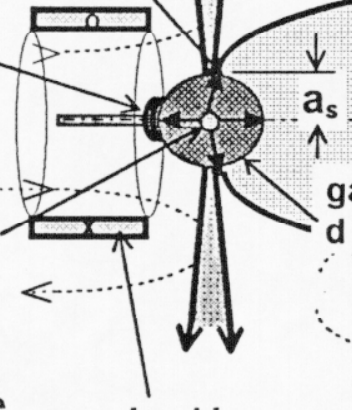

bucking solenoid

(to zero-out

fringe field)

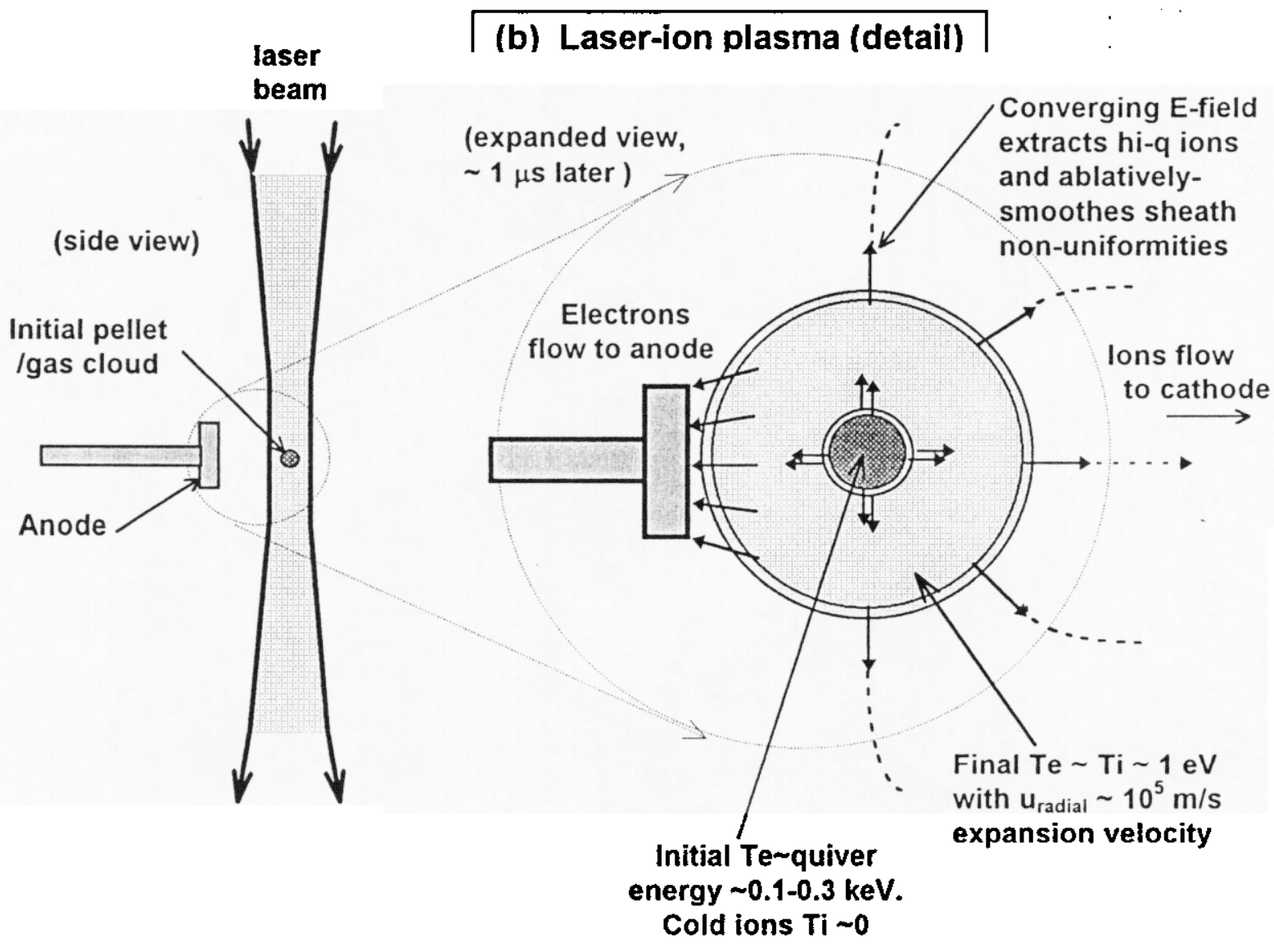

Fig. 1 Concept for a high-charge-state ion source using ultra-short-pulse lasers. 
According to applied-B diode theory [5], the strong solenoid magnetic field should prevent electrons field-emitted off the solenoid case from penetrating more than a few \% distance into the diode gap. High breakdown gradients allowed at the cathode side, together with convergence of electric field lines on the small plasma anode sheath, and a space-charge-limited beam current proportional to $(\mathrm{q} / \mathrm{A})^{1 / 2}$, are estimated to allow $100^{\prime}$ 's of amperes extracted per source. With a sufficiently high ion $q$, say $q=8$, and diode voltage $V_{3}=3 \mathrm{MV}$, ions would be injected with a sufficiently-high energy and velocity for economical magnetic transport with either solenoid or quadrupole focusing magnets, thus eliminating the need for a front-end with electrostaticquadrupole focusing arrays and beam-merging.

The main issues to be addressed for this concept in future theory and experiment (beyond the scope of this paper) are: (1) ion charge state changes by three-body recombination and by chargeexchange with ions and neutrals, and (2) ion beam brightness and emittance with beam expansion and dynamic plasma sheath motion. Use of ionization levels just below a $\mathrm{K}$ or L-shell jump should prevent beam-beam charge exchange. If the ion-neutral charge-exchange cross section were $10^{-16}$ $\mathrm{cm}^{2}$, a vacuum of $10^{-6}$ torr $\left(10^{10} \mathrm{~cm}^{-3}\right.$ neutral density) would give a mean-free path $>10 \mathrm{~km}$, much longer than the linac lengths we will consider here. But if electron capture into highly excited states resulted in cx cross sections of say, $10^{-14} \mathrm{~cm}^{2}$, a vacuum of $10^{-8}$ torr would be required.

For a given total beam energy at a given ion range required by a target, the accelerator voltage scales inversely with charge state $q$ (reducing the length of a linac for a given limit on voltage gradient), but the voltage requirement for constant ion range also decreases with lower ion mass A (in AMU), and either way of reducing the accelerator voltage proportionately raises the total beam current (or number of beams) required for the same pulse length delivered to a target. Thus aside from the source issues (1) and (2), there is the question of how much a single driver cost might be reduced with a lower ion mass $A$ at $q=1$ compared to using heavy ions at high $\mathrm{q}$, assuming laser sources could provide any ion species. And aside from the cost of a single driver, there is another question of how much economic penalty is incurred if the larger number of beams required with higher $q$ or lower $A$ were subdivided into several identical linacs (modules), so that one module could validate the cost and performance (to reduce the development cost) of a modular driver system. The remainder of this paper addresses these latter questions concerning the potential use of high-charge-state ions.

\section{Simplified cost model for modular high-q ion linacs}

A simplified cost model [6] (too lengthy to reproduce here) has been developed for linacs using laser-ion sources, and with beam transport using either superconducting solenoid or quadrupole magnets. We have applied this model consistently to compare modular driver system costs for the matrix of ion masses $A$ and charge states $q$ that are listed in Table 1 . Table 1 also gives the final ion kinetic energies $T f_{\text {ion }}$ required for a minimum ion range of $0.03 \mathrm{gm} / \mathrm{cm}^{2}$ for high gain targets. The fourth and fifth charge states are chosen to be just below an $\mathrm{L}$ or $\mathrm{K}$ shell jump, to help insure charge-state purity. Note in Table 1 the ionization levels for the highest (fifth) charge states are all about $1 \mathrm{keV}$, requiring laser intensities $\sim 10^{15} \mathrm{~W} / \mathrm{cm}^{2}$ at 0.4 micron wavelength. 
Table 1 Ion species used for a comparative cost analysis of induction linac drivers.

\begin{tabular}{|c|c|c|c|c|c|c|c|c|c|}
\hline Element & $\begin{array}{l}\text { Mass A } \\
\text { (AMU) }\end{array}$ & $\mathrm{Z}$ & $\begin{array}{l}1^{n t} \\
q\end{array}$ & $\begin{array}{c}2^{\text {nd }} \\
\mathrm{q}\end{array}$ & $\begin{array}{l}3^{\text {rd }} \\
q\end{array}$ & $\begin{array}{l}4^{\text {th }} \\
q\end{array}$ & $\begin{array}{l}5^{\text {th }} \\
q\end{array}$ & $\begin{array}{l}5^{\text {th }} E_{i} \\
(e V)\end{array}$ & $\begin{array}{c}\text { Final } \mathrm{Tf}_{\text {ion }} \\
(\mathrm{MeV})\end{array}$ \\
\hline Neon & 20 & 10 & 1 & 2 & 4 & 8 & 10 & 1362 & 151 \\
\hline Argon & 40 & 18 & 1 & 2 & 4 & 8 & 16 & 918 & 386 \\
\hline Krypton & 84 & 36 & 1 & 2 & 4 & 8 & 26 & 1206 & 1120 \\
\hline Xenon & 131 & 54 & 1 & 2 & 4 & 8 & 26 & 1013 & 2090 \\
\hline
\end{tabular}

The key constraints and assumptions used in the model of ref. [6] are listed following:

1. The laser intensity for all cases is estimated for frequency-doubled Ti:sapphire ( 0.4 micron wavelength, $100 \mathrm{fs}$ ), and scales with the desired ionization level in each case.

2. The total ion beam energy and pulse length delivered to the target is $6.5 \mathrm{MJ}$ and $10 \mathrm{~ns}$, respectively, with final kinetic energies given in Table 1 for a constant range $=0.03 \mathrm{gm} / \mathrm{cm}^{2}$.

3. The ion extraction pulse length is determined by a beam length out of the injector constrained to be less than 60 times the length on target (to have a common longitudinal spread allowance), together with a maximum laser plasma sheath expansion limited to $10 \%$ of the diode gap.

4. The diode voltage $V_{1}$ and current $I_{\text {inj }}$ are calculated for a diode size consistent with (3) and conventional vacuum breakdown scaling (minimum diode voltages), and for higher diode gradients with magnetic insulation such that $V_{s}=10 \mathrm{MV}$ (maximum diode voltage).

5. The total number of beams $\mathrm{N}_{b}$ and number of ions per source are calculated from (2) to (4).

6. The laser power and energy at $100 \mathrm{fs}$ is determined by the initial gas target size to contain the required number of ions from (5), and the intensity required to produce the charge state.

7. Each ion case is evaluated and compared for transport with either superconducting solenoids or superconducting quadrupoles, operated at the same peak field at the windings of $3 \mathrm{~T}$.

8. The beam length and average acceleration gradient $V_{\mathrm{g}}(1 \mathrm{MV} / \mathrm{m})$ are both constant. The accelerator length with "load-and-fire" gives a final energy $T_{f}$ averaged over beam length.

9. For the entire linac length, the beam radius a, transport magnet field and bore radius, and inner core radius, are all constant. The beam radius $a$ is fixed at the beginning of the magnetic transport section to match the maximum transportable current at a coil occupancy factor $\eta_{0}=$ 0.75 . For solenoids, $\eta$ is constant down the accelerator, while for quadrupoles, $\eta \sim \beta^{-1}$ as the beam accelerates. In addition, for quadrupole cases where the injection $\beta=v_{z} / c<0.023$ (corresponding to $33 \mathrm{MeV}$ Xenon), a low-cost ESQ array is inserted up to $\beta=0.023$; to shrink the magnetic transport array size at the beginning of the magnetic transport section.

10. The number of beams per linac module is seven, so that the number of modules (\# of linacs) $\mathrm{N}_{\mathrm{m}}=$ integer $\left(\mathrm{N}_{b} / 7\right)$, plus one module for any remaining number of beams less than 7 . This assumes flux return from each transport magnet is independent of adjacent beams (pessimistic). 11. Core losses are estimated as the volume of cores times $850 \mathrm{~J} / \mathrm{m}^{3}$, assuming an average pulse length of 1 microsecond, and $2.5 \mathrm{~T}$ flux swing. Pulser electrical efficiency is assumed to be 80 $\%$ based on all-solid state switching.

These simplifying assumptions allow analytic integral expressions to be evaluated for the costs of laser-ion sources at $10^{5} \$ /$ laser joule, beam transport with superconducting solenoids or quads at $\$ 50 \mathrm{~K} / \mathrm{m}$ of magnet, ferromagnetic core material at $\$ 5 / \mathrm{kg}$, pulsers at $10 \$ / \mathrm{J}$, DC/cooling at 1.5 $\$ / \mathrm{W}_{\mathrm{c}}$, and other (structure/insulators/cryo/vacuum/controls/buildings) at $\$ 50 \mathrm{~K} / \mathrm{m}$ of linac, for the various ion cases. For comparison purposes, relative costs are more useful than absolute costs with this simple model. Optimization of focusing magnet fields and core flux-swings as functions of $z$, combinations of solenoids in the front-end with more compact quads arrays in the high energy end, beam merging, etc., are all left for future work. 


\section{Results of the cost model}

Table 2 lists the minimum and maximum diode voltages for the ion cases in Table 1, and Tables 3 through 8 list, for Table 1 ion cases and for minimum and maximum diode voltages, the injector currents per beam, the injector pulse lengths, the total number of beams required for 6.5 MJ, the accelerator lengths, the normalized total driver system direct costs with solenoid transport magnets, and the normalized driver costs with quadrupole transport magnets, respectively. Table 9 summarizes different Xenon driver cases, comparing both $q=1$ with $q=26$, for both single linac drivers and modular linac drivers. All driver system costs are normalized to a reference case of a single multi-beam linac for $6.5 \mathrm{MJ}$ with $\mathrm{q}=1$ Xenon ions, an ESQ front end, and 22 beams with magnetic quadrupole transport 2100 meters long for $2.1 \mathrm{GeV}$, for which the model predicts a direct cost of $\$ 1126 \mathrm{M}$.

Table 2 Minimum and maximum injector voltages $V_{s}$ for laser-sources of ions given in Table 1 .

\begin{tabular}{r|ccccc|ccccc}
\hline & \multicolumn{4}{|c|}{ Minimum diode voltages (MV) } & \multicolumn{4}{c}{ Maximum diode voltage (MV) } \\
Element & $1^{\text {at }} \mathrm{q}$ & $2^{\text {nd }} \mathrm{q}$ & $3^{\text {rd }} \mathrm{q}$ & $4^{\text {th }} \mathrm{q}$ & $5^{\text {th }} \mathrm{q}$ & $1^{\text {ta }} \mathrm{q}$ & $2^{\text {nd }} \mathrm{q}$ & $3^{\text {rd }} \mathrm{q}$ & $4^{\text {th }} \mathrm{q}$ & $5^{\text {th }} \mathrm{q}$ \\
\hline Neon & 1.67 & 2.03 & 2.61 & 3.37 & 5.02 & 10 & 10 & 10 & 10 & 10 \\
Argon & 1.51 & 1.82 & 2.28 & 2.93 & 4.66 & 10 & 10 & 10 & 10 & 10 \\
Krypton & 1.44 & 1.72 & 2.17 & 2.77 & 5.04 & 10 & 10 & 10 & 10 & 10 \\
Xenon & 1.37 & 1.64 & 2.08 & 2.65 & 4.78 & 10 & 10 & 10 & 10 & 10 \\
\hline
\end{tabular}

Table 3 Laser-source injector currents $I_{i n j}$ at minimum and maximum $V_{\mathbf{s}}$, for Table 1 ion cases.

\begin{tabular}{|c|c|c|c|c|c|c|c|c|c|c|}
\hline \multirow[b]{2}{*}{ Element } & \multicolumn{5}{|c|}{$\mathrm{I}_{\mathrm{inj}}(\mathrm{Amps})$ at minimum diode voltages } & \multicolumn{5}{|c|}{$\mathrm{I}_{\mathrm{inj}}(\mathrm{Amps})$ at maximum diode voltage } \\
\hline & $1^{*} q$ & $2^{\text {nd }} q$ & $3^{\text {rd }} \mathrm{g}$ & $4^{\text {th }} \mathrm{g}$ & $5^{\text {th }} \mathrm{g}$ & $1^{\text {th }} \mathrm{g}$ & $2^{\text {nd }} \mathrm{g}$ & $3^{\text {rd }} \mathrm{q}$ & $4^{\text {th }} \mathrm{q}$ & $5^{\text {th }} \mathrm{q}$ \\
\hline Neon & 20 & 38 & 79 & 164 & 333 & 297 & 420 & 594 & 839 & 938 \\
\hline Argon & 12 & 23 & 46 & 94 & 267 & 210 & 297 & 420 & 594 & 839 \\
\hline Krypton & 8 & 15 & 29 & 60 & 264 & 145 & 205 & 290 & 410 & 738 \\
\hline Xenon & 6 & 11 & 22 & 45 & 195 & 116 & 164 & 232 & 328 & 591 \\
\hline
\end{tabular}

Table 4 Injector pulse lengths $\tau_{s}$ at minimum and maximum $V_{s}$, for Table 1 ion cases.

\begin{tabular}{r|ccccc|ccccc}
\hline & \multicolumn{4}{|c|}{$\tau_{\mathbf{s}}(\mu \mathrm{sec})$ at minimum diode voltages } & \multicolumn{4}{c}{$\tau_{\mathbf{s}}(\mu \mathrm{sec})$ at maximum diode voltage } \\
Element & $1^{1^{\text {ta }}} \mathrm{q}$ & $2^{\text {nd }} \mathrm{q}$ & $3^{\text {rd }} \mathrm{q}$ & $4^{\text {th }} \mathrm{q}$ & $5^{\text {th }} \mathrm{q}$ & $1^{\text {1t }} \mathrm{q}$ & $2^{\text {nd }} \mathrm{q}$ & $3^{\text {rd }} \mathrm{q}$ & $4^{\text {th }} \mathrm{q}$ & $5^{\text {th }} \mathrm{q}$ \\
\hline Neon & 5.8 & 3.7 & 2.3 & 1.4 & 1.1 & 2.4 & 1.7 & 1.2 & 0.84 & 0.8 \\
Argon & 9.7 & 6.3 & 3.9 & 2.5 & 1.4 & 3.8 & 2.7 & 1.9 & 1.3 & 0.9 \\
Krypton & 16.9 & 10.9 & 6.9 & 4.3 & 1.8 & 6.4 & 4.5 & 3.2 & 2.3 & 1.3 \\
Xenon & 23.6 & 15.2 & 9.6 & 6.0 & 2.5 & 8.7 & 6.2 & 4.4 & 3.1 & 1.7 \\
\hline
\end{tabular}


Table 5 Number of beams $\mathrm{N}_{b}$ for $6.5 \mathrm{MJ}$ at minimum and maximum $\mathrm{V}_{\mathrm{s}}$, for Table 1 ion cases. The number of 7-beam linac modules $\mathrm{N}_{\mathrm{m}}=$ integer $(\mathrm{Nb} / 7)$ plus one for any remaining beams.

\begin{tabular}{r|ccccc|ccccc}
\hline & \multicolumn{4}{|c|}{$\mathrm{N}_{\mathrm{b}}$ at minimum diode voltages } & \multicolumn{4}{c}{$\mathrm{N}_{\mathrm{b}}$ at maximum diode voltage } \\
Element & $1^{\text {th }} \mathrm{q}$ & $2^{\text {nd }} \mathrm{q}$ & $3^{\text {rd }} \mathrm{q}$ & $4^{\text {th }} \mathrm{q}$ & $5^{\text {th }} \mathrm{q}$ & $1^{\text {th }} \mathrm{q}$ & $2^{\text {nd }} \mathrm{q}$ & $3^{\text {rd }} \mathrm{q}$ & $4^{\text {th }} \mathrm{q}$ & $5^{\text {th }} \mathrm{q}$ \\
\hline Neon & 366 & 601 & 938 & 1450 & 1216 & 61 & 122 & 244 & 487 & 609 \\
Argon & 141 & 234 & 374 & 583 & 731 & 21 & 43 & 85 & 170 & 340 \\
Krypton & 44 & 73 & 116 & 181 & 323 & 6 & 13 & 25 & 50 & 163 \\
Xenon & 22 & 37 & 59 & 93 & 167 & 3 & 6 & 12 & 25 & 80 \\
\hline
\end{tabular}

Table 6 Linac lengths $L_{\mathbf{a}}$ for $1 \mathrm{MV} / \mathrm{m}$ at minimum and maximum $V_{\mathfrak{s}}$, for Table 1 ion cases.

\begin{tabular}{r|cccccc|ccccc}
\hline & \multicolumn{4}{|c|}{$\mathrm{L}_{\mathbf{a}}(\mathrm{m})$ at minimum diode voltages } & \multicolumn{4}{c}{$\mathrm{L}_{\mathbf{2}}(\mathrm{m})$ at maximum diode voltage } \\
Element & $1^{\text {a }} \mathrm{q}$ & $2^{\text {nd }} \mathrm{q}$ & $3^{\text {rd }} \mathrm{q}$ & $4^{\text {th }} \mathrm{q}$ & $5^{\text {th }} \mathrm{q}$ & $1^{\text {*t }} \mathrm{q}$ & $2^{\text {nd }} \mathrm{q}$ & $3^{\text {rd }} \mathrm{q}$ & $4^{\text {th }} \mathrm{q}$ & $5^{\text {th }} \mathrm{q}$ \\
\hline Neon & 161 & 85 & 47 & 39 & 34 & 153 & 77 & 35 & 27 & 23 \\
Argon & 397 & 204 & 107 & 58 & 44 & 388 & 196 & 99 & 41 & 35 \\
Krypton & 1131 & 572 & 292 & 152 & 55 & 1123 & 564 & 284 & 145 & 49 \\
Xenon & 2108 & 1061 & 538 & 275 & 92 & 2100 & 1053 & 530 & 268 & 87 \\
\hline
\end{tabular}

Table 7 Normalized direct costs NDC $[1=\$ 1.126 \mathrm{~B}]$ of modular $6.5 \mathrm{MJ}$ driver systems at minimum and maximum $\mathrm{V}_{\mathrm{s}}$, for Table 1 ion cases, and for solenoid transport magnets.

\begin{tabular}{r|ccccc|ccccc}
\hline & \multicolumn{4}{|c|}{ NDC at minimum diode voltages } & \multicolumn{4}{c}{ NDC at maximum diode voltage } \\
Element & $1^{\text {st }} \mathrm{q}$ & $2^{\text {th }} \mathrm{q}$ & $3^{\text {rd }} \mathrm{q}$ & $4^{\text {th }} \mathrm{q}$ & $5^{\text {th }} \mathrm{q}$ & $1^{\text {st }} \mathrm{q}$ & $2^{\text {nd }} \mathrm{q}$ & $3^{\text {rd }} \mathrm{q}$ & $4^{\text {th }} \mathrm{q}$ & $5^{\text {th }} \mathrm{q}$ \\
\hline Neon & 3.31 & 2.35 & 1.73 & 1.64 & 1.55 & 1.14 & 0.83 & 0.60 & 0.63 & 0.72 \\
Argon & 4.07 & 2.80 & 1.96 & 1.45 & 1.25 & 1.59 & 1.06 & 0.77 & 0.54 & 0.63 \\
Krypton & 5.00 & 3.33 & 2.21 & 1.51 & 0.96 & 2.10 & 1.37 & 0.96 & 0.71 & 0.56 \\
Xenon & 6.03 & 3.86 & 2.49 & 1.67 & 0.90 & 2.90 & 1.65 & 1.12 & 0.80 & 0.55 \\
\hline
\end{tabular}

Table 8 Normalized direct costs NDC $[1=\$ 1.126 \mathrm{~B}]$ of modular $6.5 \mathrm{MJ}$ driver systems at minimum and maximum $\mathrm{V}_{\mathbf{s}}$, for Table 1 ion cases, and for quadrupole transport magnets.

\begin{tabular}{r|ccccc|ccccc}
\hline & \multicolumn{4}{|c|}{ NDC at minimum diode voltages } & \multicolumn{4}{c}{ NDC at maximum diode voltage } \\
Element & $1^{\text {st }} \mathrm{q}$ & $2^{\text {nd }} \mathrm{q}$ & $3^{\text {td }} \mathrm{q}$ & $4^{\text {th }} \mathrm{q}$ & $5^{\text {th }} \mathrm{q}$ & $1^{\text {st }} \mathrm{q}$ & $2^{\text {nd }} \mathrm{q}$ & $3^{\text {rd }} \mathrm{q}$ & $4^{\text {th }} \mathrm{q}$ & $5^{\text {th }} \mathrm{q}$ \\
\hline Neon & 3.00 & 2.03 & 1.31 & 1.29 & 1.33 & 0.67 & 0.57 & 0.48 & 0.53 & 0.64 \\
Argon & 2.58 & 2.37 & 1.48 & 1.10 & 1.02 & 0.82 & 0.63 & 0.52 & 0.43 & 0.53 \\
Krypton & 2.23 & 1.96 & 1.71 & 1.05 & 0.79 & 1.12 & 0.68 & 0.56 & 0.48 & 0.46 \\
Xenon & 2.26 & 1.86 & 1.60 & 1.26 & 0.70 & 1.30 & 0.89 & 0.58 & 0.49 & 0.42 \\
\hline
\end{tabular}


Table 9 Comparison of $6.5 \mathrm{MJ}$ driver systems with minimum $\mathrm{V}_{\mathrm{a}}$, Xenon with $2.1 \mathrm{GeV}$ final ion energy for $0.03 \mathrm{gm} / \mathrm{cm}^{2}$ ion range in a target, and quadrupole transport magnets.

\begin{tabular}{r|cccc}
\hline Parameter & $\begin{array}{c}\text { Single linac } \\
\left(\mathrm{Xe}^{+1}\right)\end{array}$ & $\begin{array}{c}\text { Modular linacs } \\
\left(\mathrm{Xe}^{+1}\right)\end{array}$ & $\begin{array}{c}\text { Single linac } \\
\left(\mathrm{Xe}^{+26}\right)\end{array}$ & $\begin{array}{c}\text { Modular linacs } \\
\left(\mathrm{Xe}^{+26}\right)\end{array}$ \\
\hline \# of beams $\left(\mathrm{N}_{\mathrm{b}}\right)$ & 22 & 22 & 167 & 167 \\
\# of linacs $\left(\mathrm{N}_{\mathrm{m}}\right)$ & 1 & $3(7-$ beam $)+1$ & 1 & 23 (7-beam $)+1$ \\
Length $(\mathrm{m})$ & 2108 & 2108 & 92 & 92 \\
Core radius $(\mathrm{m})$ & 0.87 & 0.51 & 3.18 & 0.69 \\
Efficiency $(\%)$ & 33 & 16 & 72 & 46 \\
Normalized cost & 1 & 2.26 & 0.38 & 0.70 \\
\hline
\end{tabular}

\section{Conclusions}

At this point many technical issues remain concerning the laser-ion-source concept shown in Fig.1 that will require detailed simulations and experiments to resolve, including high-q ion recombination and charge-exchange losses, limits on magnetized diode electric field gradient, extracted beam emittance from an expanding plasma sheath, longitudinal beam confinement and drift compression, and beam charge neutralization in the target chamber, to name a few. The question addressed here is: assuming laser-ion sources would work as modeled (or any equivalent high-q ion source), would there be sufficient potential benefits for a future driver, to justify the further $R \& D$ required for such sources? Based on different ion cases compared using a simple but consistently-applied cost model, the answer is: the potential benefits of high-q ion sources might be very large for drivers, and with important implications for the development cost of ion drivers. Quantitatively, this work suggests the potential benefits are: for single linac drivers, reduced driver cost (by $2.6 \mathrm{x}$ ) and increased efficiency (by $2.2 \mathrm{x}$ ), and for a modular driver system of linacs, by factors of 3.2 and 2.9, respectively. Most important, use of high-q ions for modular architectures might dramatically reduce the cost of an accelerator prototype which could both fully validate the performance of a driver, while providing significant capability for target physics experiments. If the simple cost model used here was to be trusted, one module of the $\mathrm{Xe}^{+26}$ system described in Table 9 would deliver $6.5 / 24 \sim 0.27 \mathrm{MJ}$ of $2 \mathrm{GeV}$ ions at a cost $\sim \mathrm{fx} 0.7 \mathrm{x}$ $1126 / 24 \sim \$ 33 \mathrm{Mxf}$, where $\mathrm{f}$ is some cost multiplier $>1$ for a one-of-a-kind prototype cost, as opposed to a mature driver system of modules. Conclusion? We should do more research on high-q ion sources.

*Work performed under the auspices of the US Department of Energy by Lawrence Livermore National Laboratory under Contract W-7405-Eng-48

\section{References}

[1] D. J. Dudziak, W. W. Saylor, and W. B. Herrmannsfeldt, "U.S. Heavy-Ion Fusion Systems Assessment Project Overview", Fusion Technology, Vol. 13, p. 207, Feb. 1988

[2] Laser and Particle Beams (1966), Vol. 14, No.3, see in particular, I. V. Roudsky "General features of highly charged ion generation in laser-produced plasmas", p. 369.

[3] D. A. Callahan, "Chamber propagation physics for heavy ion fusion", 7th Int. Symposium on Heavy-Ion Inertial Fusion, in Fusion Engineering and Design 32-33, (Nov. 1996), p.441

[4] M. D. Perry, A. Szoke, O. L. Landen, and E. M. Campbell, "Nonresonant Multiphoton Ionization of Noble Gases: Theory and Experiment", Phys. Rev. Letters, 60, No.13, Mar. 1988, p. 1270. See also M. D. Perry, et. al., Optical Society of Am. B, Vol.6, No. 3, Mar. 1989, p. 344 [5] M. P. Desjarlais, "Theory of Applied-B Ion Diodes", Phys. Fluids B 1(8), Aug. 1989, p. 1709 
[6] B. G. Logan, M. D. Perry, and G. J. Caporaso "Model for High-Charge-State Ion Induction Accelerators", Lawrence Livermore National Laboratory report UCRL-ID-126815, Feb. 1997.

8 


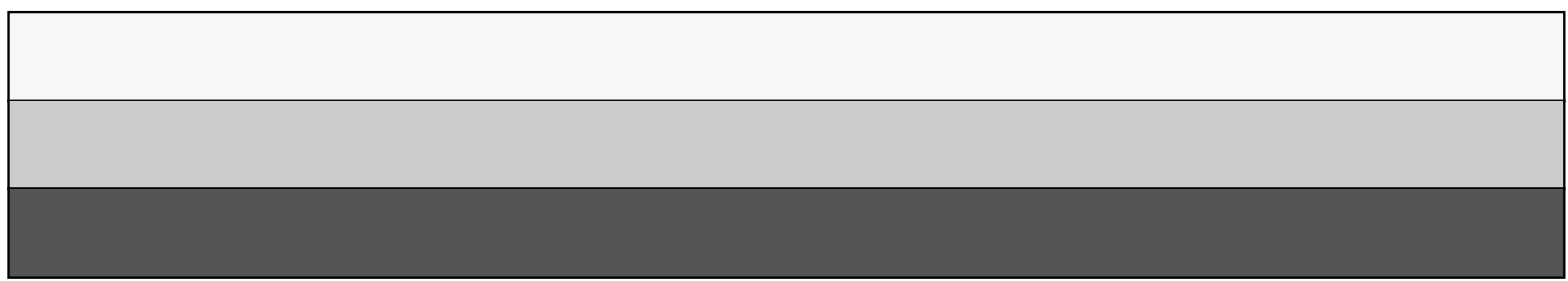

